\title{
Electron Tomography of Hydroxyapatite Platelets in Atractosteus spatula Boney Scales
}

Kenneth J.T. Livi ${ }^{1}$, Trevor Lancon ${ }^{2}$ and Cedric Bouchet-Marquis ${ }^{3}$

1. Dept. of Materials Science and Engineering, Johns Hopkins University, Baltimore MD 21218 USA

2. Thermo Fisher Scientific, Houston, TX 77084 USA

3. Thermo Fisher Scientific, Hillsboro, OR 97124 USA

Although the structural scaffolding of bone is type I collagen, the stiffening agent is inorganic calciumorthophosphate or hydroxyapatite (HAp). The physical properties of bone are greatly affected by the three dimensional arrangement of HAp within collagen fibrils and fibers. Thus, many studies have been designed to determine the relationship between HAp and the collagen molecules [1]. It has long been known that HAp crystallizes within bone collagen as poorly crystalline material in young bone and matures into larger platelets with age. However, while the density of packing of HAp within bone has led to the discovery of the overall orientation of crystals through X-ray diffraction techniques, it has hampered the observation of individual platelet shapes and their intimate relationships with collagen by electron microscopy.

We present data from dual axis electron tomography tilt series that allow reconstructions of individual HAp platelets within sections made from alligator gar fish (Atractosteus spatula) boney scales. The tilt series were acquired from sections that were stained with $\mathrm{OsO}_{4}$ and partially demineralized. Demineralization occurred along fronts that created regions that were depopulated of HAp crystals, and the $\mathrm{OsO}_{4}$ allowed for the overlap-gap band structure of the collagen fibrils to be imaged. Thus, isolated individual HAp crystals could be imaged in situ relative to the collagen fibril axis.

The tilt series data were collected not using STEM HAADF, but in wide beam conditions. Thus, the contrast within the sections was mainly due to diffraction scattering. Since HAp in bone is known to crystallize as extremely thin platelets, dark contrast is only created when the platelets are in strong diffraction conditions (SDC). This occurs when the platelet basal plane is parallel to the incident beam. In the early days of TEM observations of bone, HAp was thought to be in the shape of a needle because only lines of dark contrast were observed. However, upon tilting of the section, the dark lines would shift sideways, indicative of a curved sheet, as opposed to remaining stationary as a needle would. The orientation of SDC is also where crystals would be thickest in projection and would appear darkest in images solely relying on mass thickness. Thus, diffraction contrast, in the case of extremely thin crystals, augments mass-thickness contrast and can be used to reconstruct crystal shapes.

Figure 1 presents the conventional image of gar scale bone at the demineralization front. Visible are the ordered bands of stained collagen (67 nm repeat)(long arrows) and long thin dark lines of HAp crystals in SDC (short arrows). Also in the image are HAp crystals with their basal normals parallel to the electron beam (starred). The sparseness of HAp crystals allows for the unambiguous reconstruction of individual crystals, one of which is presented in Figure 2. The roughness of the surface of the platelet is likely a consequence of $\mathrm{OsO}_{4}$ attaching to the surface of the HAp. The true extent of the surface roughness is not known. Important features of the reconstructions are that the HAp platelets are curved, are found at an angle to the collagen long axis, and that the length of the crystals can be much greater than the $67 \mathrm{~nm}$ band repeat. It has been hypothesized that, at least in the early stages of bone growth, 
HAp was confined to the gap region of the collagen $(\sim 40 \mathrm{~nm})$. However, in mature bone, the HAp crystals extend beyond the $67 \mathrm{~nm}$ repeat. Additional STEM HAADF data confirms the size and angled orientation $\left(\sim 10^{\circ}\right)$ of HAp relative to the collagen axis. The consequence of this rotation is that collagen and HAp must interpenetrate each other. This would have a profound effect on the stiffness of the composite. These observations call for a revised model for bone.

\section{References:}

[1] M Glimcher in "Medical Mineralogy and Geochemistry", RiMG Vol 64, eds. N Sahai \& M Schoonen, (Mineralogical Society America), p. 223.

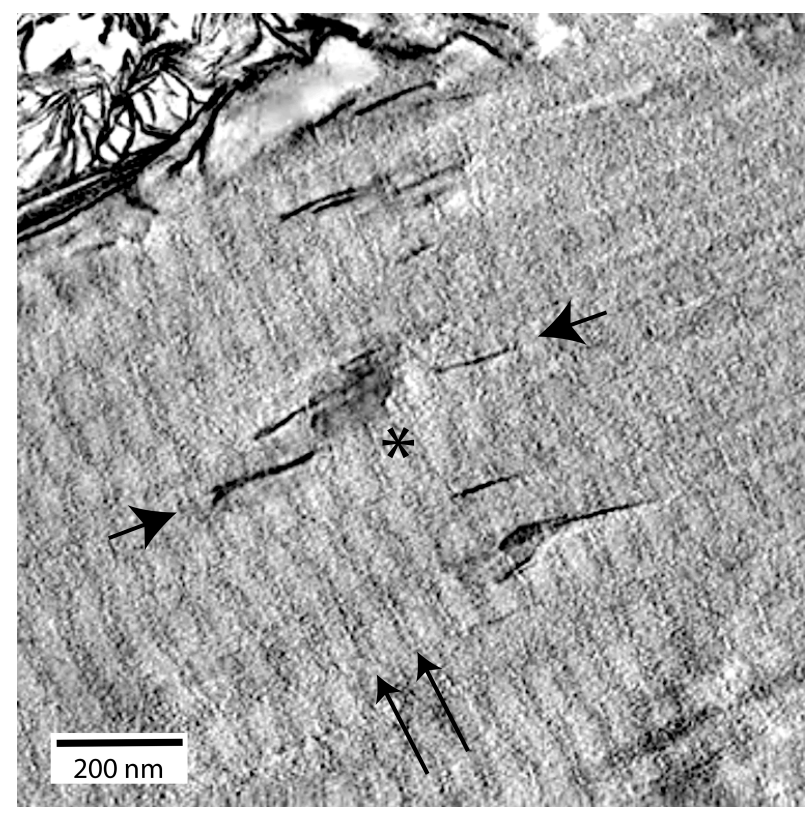

Figure 1. Partially demineralized, $\mathrm{OsO}_{4}$ stained section of bone showing the collagen banding structure (long arrows) and individual HAp crystals (short arrows)

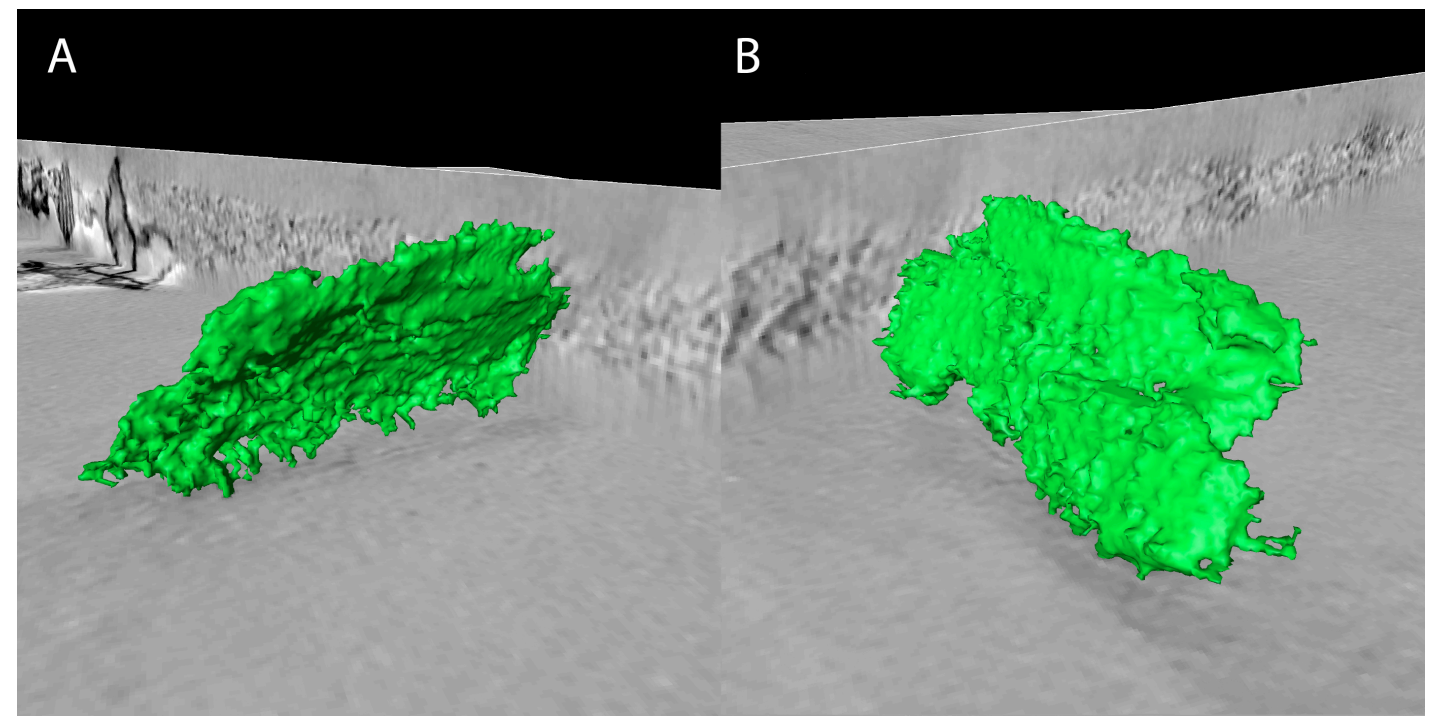

Figure 2. Reconstruction of a single HAp crystal. 